ISSN 1855-3966 (printed edn.), ISSN 1855-3974 (electronic edn.)

\author{
ARS MATHEMATICA CONTEMPORANEA 17 (2019) 455-466 \\ https://doi.org/10.26493/1855-3974.1925.57a \\ (Also available at http://amc-journal.eu)
}

\title{
Unicyclic graphs with the maximal value of Graovac-Pisanski index*
}

\author{
Martin Knor \\ Slovak University of Technology in Bratislava, Faculty of Civil Engineering, \\ Department of Mathematics, Bratislava, Slovakia
}

Jozef Komorník

Faculty of Management, Comenius University, Odbojárov 10, P.O. Box 95, Bratislava, Slovakia

Riste Škrekovski

Faculty of Information Studies, Novo mesto and FMF, University of Ljubljana, Slovenia

Aleksandra Tepeh

Faculty of Information Studies, Novo mesto and

Faculty of Electrical Engineering and Computer Science, University of Maribor, Slovenia

Received 31 January 2019, accepted 13 May 2019, published online 8 November 2019

\begin{abstract}
Let $G$ be a graph and let $\Gamma$ be its group of automorphisms. Graovac-Pisanski index of $G$ is $\operatorname{GP}(G)=\frac{|V(G)|}{2|\Gamma|} \sum_{u \in V(G)} \sum_{\alpha \in \Gamma} d(u, \alpha(u))$, where $d(u, v)$ is the distance from $u$ to $v$ in $G$. One can observe that $\operatorname{GP}(G)=0$ if $G$ has no nontrivial automorphisms, but it is not known which graphs attain the maximum value of Graovac-Pisanski index. In this paper we show that among unicyclic graphs on $n$ vertices the $n$-cycle attains the maximum value of Graovac-Pisanski index.
\end{abstract}

Keywords: Graovac-Pisanski index, modified Wiener index, unicyclic graphs.

Math. Subj. Class.: 05C25

* The first author acknowledges partial support by Slovak research grants VEGA 1/0142/17, VEGA 1/0238/19, APVV-15-0220 and APVV-17-0428. The research was partially supported also by Slovenian research agency ARRS, program no. P1-00383, projects no. L1-4291 and J1-1692.

E-mail addresses: knor@math.sk (Martin Knor), jozef.komornik@fm.uniba.sk (Jozef Komorník), skrekovski@gmail.com (Riste Škrekovski), aleksandra.tepeh@gmail.com (Aleksandra Tepeh)

()(7) This work is licensed under https://creativecommons.org/licenses/by/4.0/ 


\section{Introduction}

Wiener index, the sum of distances in a graph, is an important molecular descriptor. It was introduced by Wiener in 1949, see [18], and since then many other molecular descriptor have appeared. One of them is the Graovac-Pisanski index [8], originally known as the modified Wiener index. With this index an algebraic approach for generalizing the Wiener index was presented. Namely, as the Wiener index also the Graovac-Pisanski index is based on distances but its advantage is in considering also the symmetries of a graph, and it is known that symmetries of a molecule have an influence on its properties [14].

In his pioneering paper, Wiener showed a correlation of the Wiener index of alkanes with their boiling points [18]. It turns out that the Graovac-Pisanski index combines the symmetry and topology of molecules to obtain a good correlation with some physicochemical properties of molecules. Recently, Črepnjak et al. showed that the GraovacPisanski index of some hydrocarbon molecules is correlated with their melting points [6].

This index also drew attention from theoretical point of view. Researchers are interested in the difference between the Wiener and Graovac-Pisanski index. This difference was computed in [9] for some families of polyhedral graphs. The Graovac-Pisanski index of nanostructures was studied in $[1,2,15,16,17]$ and for some classes of fullerenes and fullerene-like molecules in [3, 11, 12]. In [13] the symmetry groups and Graovac-Pisanski index of some linear polymers were computed. Upper and lower bounds for GraovacPisanski index were considered in [11]. In [7] and [16] Graovac-Pisanski index was further considered from computational point of view. Exact formulae for the Graovac-Pisanski index for some graph operations are presented in [4]. Recently it was proved that for any connected bipartite graph, as well as for any connected graph on even number of vertices, the Graovac-Pisanski index is an integer number [5].

Let $G$ be a connected graph. The Graovac-Pisanski index of $G$ is defined as

$$
\operatorname{GP}(G)=\frac{|V(G)|}{2|\operatorname{Aut}(G)|} \sum_{u \in V(G)} \sum_{\alpha \in \operatorname{Aut}(G)} \operatorname{dist}_{G}(u, \alpha(u)),
$$

where $\operatorname{Aut}(G)$ is the group of automorphisms of $G$ and $\operatorname{dist}_{G}(u, v)$ denotes the distance from $u$ to $v$ in $G$. However, in the paper we will use a result from [5] to compute this index. To explain the method we need some additional definitions. Let $G$ be a graph, $u \in V(G)$ and $S \subseteq V(G)$. The distance of $u$ in $S, w_{S}(u)$, is defined as

$$
w_{S}(u)=\sum_{v \in S} \operatorname{dist}_{G}(u, v)
$$

The group of automorphisms of $G$ partitions $V(G)$ into orbits. We say that $u, v \in V(G)$ belong to the same orbit if there is an automorphism $\alpha \in \operatorname{Aut}(G) \operatorname{such}$ that $\alpha(u)=v$. Let $V_{1}, V_{2}, \ldots, V_{t}$ be all the orbits of $\operatorname{Aut}(G)$ in $G$. Moreover, for every $i, 1 \leq i \leq t$, let $v_{i} \in V_{i}$. That is, $v_{i}$ 's are the representatives of $V_{i}$ 's. It was shown in [5] that

$$
\operatorname{GP}(G)=\frac{|V(G)|}{2} \sum_{i=1}^{t} w_{V_{i}}\left(v_{i}\right) .
$$

By (1.1), if a graph has no nontrivial automorphisms, that is if all its orbits consist of single vertices, then its Graovac-Pisanski index is 0 . Hence, all graphs with no nontrivial automorphisms achieve the minimum value of Graovac-Pisanski index. More interesting is the opposite problem. 
Problem 1.1. Find all graphs on $n$ vertices with the maximum value of Graovac-Pisanski index.

This problem was solved for trees in [10]. By a long $H$ on $n$ vertices we denote a tree obtained from a path on $n-4$ vertices by attaching two pendent vertices to each endvertex of the path.

Theorem 1.2. Let $T$ be a tree on $n \geq 8$ vertices with the maximum value of GraovacPisanski index. Then $T$ is either a path or a long H. Moreover,

$$
\operatorname{GP}(T)= \begin{cases}\frac{n^{3}-n}{8} & \text { if } n \text { is odd } \\ \frac{n^{3}}{8} & \text { if } n \text { is even }\end{cases}
$$

For $n \leq 7$ there are also three other trees with the maximum value of Graovac-Pisanski index. However, they have the value of Graovac-Pisanski index as stated in Theorem 1.2.

In this paper we prove the following statement.

Theorem 1.3. Let $G$ be a unicyclic graph on $n$ vertices with the maximum value of GraovacPisanski index. Then $G$ is the $n$-cycle and

$$
\operatorname{GP}\left(C_{n}\right)= \begin{cases}\frac{n^{3}-n}{8} & \text { if } n \text { is odd, } \\ \frac{n^{3}}{8} & \text { if } n \text { is even. }\end{cases}
$$

Observe that Graovac-Pisanski index for extremal trees and for extremal unicyclic graphs has the same value. We believe the following holds.

Conjecture 1.4. Let $G$ be a graph on $n$ vertices, $n \geq 8$, with the maximum value of Graovac-Pisanski index. Then $G$ is either a path, or a long $H$, or a cycle.

To support this conjecture we performed some computer experiments. They showed the validity of the conjecture for $n=8$ and $n=9$. We believe that the maximal degree of extremal graphs is small (at most 3), thus for the cases $n=10$ and $n=11$ we limited our computer search to maximal degrees 5 and 4 , respectively, and in these cases the conjecture was confirmed as well. The graphs from the conjecture are extremal also for $n \in\{5,6,7\}$, however when $n$ equals 7 there exists an additional extremal graph.

\section{Proof}

In this section we prove several claims which imply Theorem 1.3. Obviously, if we consider graphs of order $n$, we do not need to consider the multiplicative term $\frac{n}{2}$ in (1.1). Therefore we define

$$
\operatorname{GPa}(G)=\sum_{i=1}^{t} w_{V_{i}}\left(v_{i}\right),
$$

where $V_{1}, V_{2}, \ldots, V_{t}$ are all the orbits of $\operatorname{Aut}(G)$ in $G$ and $v_{1}, v_{2}, \ldots, v_{t}$ are their representatives, respectively. Then for given $n$, graphs on $n$ vertices with the maximum value of GPa are the solutions of Problem 1.1.

For a cycle on $n$ vertices, $\operatorname{GPa}\left(C_{n}\right)=w_{V}(v)$ where $v$ is an arbitrary vertex of $C_{n}$ and $V=V\left(C_{n}\right)$. This implies the following statement. 
Proposition 2.1. We have

$$
\operatorname{GPa}\left(C_{n}\right)= \begin{cases}\frac{n^{2}-1}{4} & \text { if } n \text { is odd, } \\ \frac{n^{2}}{4} & \text { if } n \text { is even }\end{cases}
$$

In what follows we generalize the GPa-parameter. Let $\mathcal{Z}=\left\{Z_{1}, \ldots, Z_{t_{\mathcal{Z}}}\right\}$ be a partition of $V(G)$ and let $z_{i} \in Z_{i}, 1 \leq i \leq t_{\mathcal{Z}}$. Then

$$
\operatorname{GPa}^{\mathcal{Z}}(G)=\sum_{i=1}^{t_{\mathcal{Z}}} w_{Z_{i}}\left(z_{i}\right)
$$

In our proofs, sets $Z_{i}$ will usually be unions of orbits of $\operatorname{Aut}(G)$. Nevertheless, $\operatorname{GPa}^{\mathcal{Z}}(G)$ will depend on the choice of the representatives $z_{i}$.

To prove Theorem 1.3 we start with $\operatorname{GPa}(G)$, where $G$ is an extremal unicyclic graph on $n$ vertices different from the $n$-cycle. Then in a sequence of steps we modify either the graph or the partition and in each step we obtain a larger value of $\mathrm{GPa}^{\mathcal{Z}}$. Since we terminate this process with $C_{n}$ and GPa, we get the result.

Hence, let $G$ be a unicyclic graph on $n$ vertices with the maximum value of GraovacPisanski index and such that $G$ is not the $n$-cycle. Then $G$ consists of a single cycle $C$ and trees rooted at the vertices of the cycle. In what follows, orbits of vertices of $C$ will be important.

We start with modifying the partition by merging together some orbits of vertices which have the same distance from $C$. We denote by $\mathcal{X}$ the new partition of $V(G)$, while the original partition into orbits is denoted by $\mathcal{V}$. Let $v$ be a vertex of $C$. If $\{v\}$ is a trivial orbit of $\operatorname{Aut}(G)$, then orbits in the $v$-rooted tree form sets of the partition $\mathcal{X}$. But if $\{v\}$ is not a trivial orbit of $\operatorname{Aut}(G)$, we do the following. Let $O_{v}$ be the orbit of $\operatorname{Aut}(G)$ containig $v$ and let $O_{v}(G)$ be the set of vertices of $u$-rooted trees where $u \in O_{v}$. We partition the vertices of $O_{v}(G)$ according to their distance from $C$. Hence, $O_{v}$ alone is one set of $\mathcal{X}$, another set of $\mathcal{X}$ contains those vertices of $O_{v}(G)$ which are adjacent to a vertex of $C$, etc. We have the following statement.

Lemma 2.2. For arbitrary choice of the representatives of sets in $\mathcal{X}$ we have

$$
\mathrm{GPa}(G) \leq \operatorname{GPa}^{\mathcal{X}}(G) .
$$

Proof. Let $X_{i}$ be a set from $\mathcal{X}$ and let $x_{i}$ be an abitrary vertex of $X_{i}$. Observe that $X_{i}$ is a union of several orbits of $\operatorname{Aut}(G)$. Let $V_{0}$ be an orbit of $\operatorname{Aut}(G)$ such that $V_{0} \subseteq X_{i}$. Then $w_{V_{0}}(u)$ is the same for every $u \in V_{0}$. So let $v_{0}$ be a vertex of $V_{0}$ at the shortest distance from $x_{i}$. Then both $x_{i}$ and $v_{0}$ are in the same tree rooted at a vertex of $C$. Assume that they are in a $v$-rooted tree $T$.

Let $u$ be a vertex of $V_{0}$. If $u$ is not in $T$ then $\operatorname{dist}_{G}\left(x_{i}, u\right)=\operatorname{dist}_{G}\left(v_{0}, u\right)$ since $\operatorname{dist}_{G}\left(x_{i}, v\right)=\operatorname{dist}_{G}\left(v_{0}, v\right)$. So let $u$ be a vertex in $T$. Let $z$ be a vertex on the (unique) $\left(v_{0}, u\right)$-path at the shortest distance from $v$. Since $v_{0}$ is a vertex of $V_{0}$ at the shortest distance from $x_{i}$, the shortest $\left(x_{i}, u\right)$-path must contain $z$. Thus $\operatorname{dist}_{G}\left(v_{0}, u\right) \leq \operatorname{dist}_{G}\left(x_{i}, u\right)$ and so $w_{V_{0}}\left(v_{0}\right) \leq w_{V_{0}}\left(x_{i}\right)$. Consequently, $\operatorname{GPa}(G) \leq \operatorname{GPa}^{\mathcal{X}}(G)$ as required.

Now we modify the graph $G$, and we consider a partition $\mathcal{Y}$ of the vertex set of the modified graph inherited from the partition $\mathcal{X}$ of $G$. So let $v$ be a vertex of $C$. If $\{v\}$ is a 
trivial orbit of $\operatorname{Aut}(G)$ then we do not change the $v$-rooted tree, and its orbits form sets of the partition $\mathcal{Y}$. Hence, in this case the sets of $\mathcal{Y}$ coincide with the sets of $\mathcal{X}$ (and also with the orbits of $\mathcal{V}$ ). But if $\{v\}$ is not a trivial orbit of $\operatorname{Aut}(G)$ then we change the $v$-rooted tree. If the $v$-rooted tree has $p$ vertices in $G$ then we replace it by a path on $p$ vertices rooted at the endvertex, which we again denote by $v$. Denote by $F$ the graph which results when all these replacements are made. Since we did not change the cycle, we denote the cycle of $F$ again by $C$. Let $O_{v}$ be the orbit of $\operatorname{Aut}(G)$ containing $v$. By our assumption $\left|O_{v}\right| \geq 2$. Analogously as above, let $O_{v}(F)$ be the set of vertices of $u$-rooted trees where $u \in O_{v}$. Partition $O_{v}(F)$ into $p$ disjoint sets of $\mathcal{Y}$ according to their distance from $C$.

Observe that for every $Y_{i} \in \mathcal{Y}$ and for every two vertices $y_{i}^{1}, y_{i}^{2} \in Y_{i}$ we have $w_{Y_{i}}\left(y_{i}^{1}\right)=w_{Y_{i}}\left(y_{i}^{2}\right)$. Hence when computing $\mathrm{GPa}^{\mathcal{Y}}(F)$, we can choose the representatives $y_{i}$ in $Y_{i}$ arbitrarily. However, orbits of $F$ may be strictly larger than the sets $Y_{i}$. This is caused by the fact that two non-isomorphic rooted trees may have the same numbers of vertices. Our next statement follows.

Lemma 2.3. For arbitrary choice of representatives of sets in $\mathcal{Y}$ we have

$$
\operatorname{GPa}^{\mathcal{X}}(G) \leq \operatorname{GPa}^{\mathcal{Y}}(F) .
$$

Proof. Let $H$ be a graph. A ray in $H$ is a subgraph of $H$ which is isomorphic to a path, its first vertex has degree at least 3 in $H$, its last vertex has degree 1 in $H$ and all the other vertices have degree 2 in $H$.

We do not prove the inequality directly. Instead, we construct a sequence of graphs $G=G^{0}, G^{1}, \ldots, G^{q}=F$, each $G^{i}$ with a partition $\mathcal{X}^{i}$, such that

$$
\operatorname{GPa}^{\mathcal{X}^{i}}\left(G^{i}\right) \leq \mathrm{GPa}^{\mathcal{X}^{i+i}}\left(G^{i+1}\right)
$$

for a special choice of representatives in $\mathcal{X}^{i+1}$, where $0 \leq i \leq q-1, \mathcal{X}^{0}=\mathcal{X}$ and $\mathcal{X}^{q}=\mathcal{Y}$. We remark that for every $i, G^{i}$ will be a unicyclic graph with the cycle $C$ such that if $O=$ $\left\{v_{1}, \ldots, v_{t}\right\}$ is an orbit of vertices of $C$ in $G$, then all $v_{j}$-rooted trees in $G^{i}$ are mutually isomorphic, $1 \leq j \leq t$. If $t=1$ then the $v_{1}$-rooted trees in $G, G^{1}, \ldots, F$ are mutually isomorphic and all $\mathcal{X}, \mathcal{X}^{1}, \ldots, \mathcal{Y}$ coincide on the vertex sets of these trees. However if $t \geq 2$, then the vertex set $O_{v_{1}}\left(G^{i}\right)$ of the $v_{j}$-rooted trees, $1 \leq j \leq t$, is partitioned in $\mathcal{X}^{i}$ according to the distance from $C$, and we assume that all the representatives of these sets are in the $v_{1}$-rooted tree. This assupmtion is possible since $O$ is an orbit in $G$, and although $O$ does not need to be an orbit of $G^{i}$, the vertices of $O$ are nicely distributed along the cycle $C$ in $G^{i}$.

So consider $i, 0 \leq i<q$. We assume that $G^{i}$ is already known and we construct $G^{i+1}$. For this, let $O=\left\{v_{1}, \ldots, v_{t}\right\}$ be an orbit of vertices of $C$ in $G$, where $t \geq 2$. If the $v_{1}$ rooted tree (and so also $v_{j}$-rooted trees for $2 \leq j \leq t$ ) is a path rooted at the endvertex, then we are done with this orbit of $G$. So suppose that the $v_{1}$-rooted tree has at least two endvertices different from $v_{1}$, and consequently, at least two distinct rays starting at a common vertex. Let $R_{1}$ and $R_{2}$ be two rays starting at a vertex $c$ such that $\operatorname{dist}_{G^{i}}\left(v_{1}, c\right)$ is maximum possible. We assume that $R_{1}$ is not shorter than $R_{2}$. If there is a representative $x_{j}^{i}$ of $X_{j}^{i}$ which is in $R_{2}$, then replace it by a vertex of $X_{j}^{i}$ in $R_{1}$. Observe that this replacement does not change $\mathrm{GPa}^{\mathcal{X}^{i}}\left(G^{i}\right)$. Now delete $R_{2}$ from the $v_{1}$-rooted tree and attach it to the second vertex of $R_{1}$. Moreover, repeat the same procedure in all the other $v_{j}$-rooted trees, $2 \leq j \leq t$, and denote by $G^{i+1}$ the resulting graph. Denote by $T^{i}$ and $T^{i+1}$ the $v_{1}$-rooted 
tree in $G^{i}$ and $G^{i+1}$, respectively. If $R_{1}$ and $R_{2}$ have the same length, then this operation may create a new set in $\mathcal{X}^{i+1}$, because $T^{i}$ may have smaller depth than $T^{i+1}$. (As is the custom, by depth we denote the largest distance from the root.) In such a case choose a representative of this new set in $R_{2}$. This is the unique case when a representative will be in $R_{2}$ in $T^{i+1}$.

Let $d=\operatorname{dist}_{G^{i}}\left(v_{1}, c\right)$ and let $\ell$ be the length of $R_{2}$. Assume that the indices of sets in $\mathcal{X}^{i}$ and $\mathcal{X}^{i+1}$ are chosen so that $X_{j}^{i+1}$ in $G^{i+1}$ was obtained from $X_{j}^{i}$ in $G^{i}$ and the representatives of $X_{j}^{i}$ and $X_{j}^{i+1}$ coincide whenever possible. Then $w_{X_{j}^{i}}\left(x_{j}^{i}\right)$ in $G^{i}$ may differ from $w_{X_{j}^{i+1}}\left(x_{j}^{i+1}\right)$ in $G^{i+1}$ only if $X_{j}^{i+1}$ contains vertices of $v_{k}$-rooted trees, $1 \leq$ $k \leq t$, which are at distance $d+1, d+2, \ldots, d+\ell+1$ from $C$. We distinguish three cases.

Case 1: $X_{j}^{i+1}$ contains vertices at distance $d+1$ from $C$. Then in the $v_{1}$-rooted tree, $X_{j}^{i+1}$ is smaller than $X_{j}^{i}$ by exactly one vertex. Consequently $\left|X_{j}^{i}\right|-\left|X_{j}^{i+1}\right|=t$. Comparing to $\mathrm{GPa}^{\mathcal{X}^{i}}\left(G^{i}\right), \mathrm{GPa}^{\mathcal{X}^{i+1}}\left(G^{i+1}\right)$ is decreased by 2 due to a missing vertex in $T^{i+1}$ and it is decreased by $(t-1) 2(d+1)+c$ due to missing vertices in $v_{k}$-rooted trees $2 \leq k \leq t$. Here $c$ represents the distances using the edges of $C$, that is $c=\sum_{k=2}^{t} \operatorname{dist}_{G}\left(v_{1}, v_{k}\right)$. Hence,

$$
w_{X_{j}^{i+1}}\left(x_{j}^{i+1}\right)-w_{X_{j}^{i}}\left(x_{j}^{i}\right)=-2-(t-1) 2(d+1)-c .
$$

Case 2: $X_{j}^{i+1}$ contains vertices at distance $d+a$ from $C$, where $2 \leq a \leq \ell$. Then $\left|X_{j}^{i+1}\right|=\left|X_{j}^{i}\right|$ and comparing to $\mathrm{GPa}^{\mathcal{X}^{i}}\left(G^{i}\right), \mathrm{GPa}^{\mathcal{X}^{i+1}}\left(G^{i+1}\right)$, is decreased by 2 due to a shorter distance to a vertex of $X_{j}^{i+1}$ in $R_{2}$. Hence,

$$
w_{X_{j}^{i+1}}\left(x_{j}^{i+1}\right)-w_{X_{j}^{i}}\left(x_{j}^{i}\right)=-2 .
$$

Case 3: $X_{j}^{i+1}$ contains vertices at distance $d+\ell+1$ from $C$. Then in the $v_{1}$-rooted tree, $X_{j}^{i+1}$ is larger than $X_{j}^{i}$ by exactly one vertex. Consequently $\left|X_{j}^{i+1}\right|-\left|X_{j}^{i}\right|=t$. Comparing to $\mathrm{GPa}^{\mathcal{X}^{i}}\left(G^{i}\right), \mathrm{GPa}^{\mathcal{X}^{i+1}}\left(G^{i+1}\right)$ is increased by $(t-1) 2(d+\ell+1)+c$ due to new vertices in $v_{k}$-rooted trees, $2 \leq k \leq t$. Here $c$ is the very same constant as in Case 1 , that is $c=\sum_{k=2}^{t} \operatorname{dist}_{G}\left(v_{1}, v_{k}\right)$. In some cases, namely if $X_{j}^{i}$ is not empty, $\mathrm{GPa}^{\mathcal{X}^{i}}$ is increased by at least 2 due to a new vertex in $T^{i+1}$, but we do not need to consider this contribution in our calculations. Hence,

$$
w_{X_{j}^{i+1}}\left(x_{j}^{i+1}\right)-w_{X_{j}^{i}}\left(x_{j}^{i}\right) \geq(t-1) 2(d+\ell+1)+c .
$$

Since $w_{X_{j}^{i+1}}\left(x_{j}^{i+1}\right)=w_{X_{j}^{i}}\left(x_{j}^{i}\right)$ when $X_{j}^{i} \nsubseteq \subseteq O_{v_{i}}\left(G^{i}\right)$, summing the expressions (2.2), (2.3) and (2.4) we get

$$
\begin{aligned}
\operatorname{GPa}^{\mathcal{X}^{i+1}}\left(G^{i+1}\right)-\mathrm{GPa}^{\mathcal{X}^{i}}\left(G^{i}\right) \geq & (-2-(t-1) 2(d+1)-c)-(\ell-1) 2 \\
& +((t-1) 2(d+\ell+1)+c) \\
= & (t-2) 2 \ell \geq 0
\end{aligned}
$$

since $t \geq 2$.

Let $Y_{i} \in \mathcal{Y}$. Observe that if $Y_{i} \cap V(C) \neq \emptyset$, then $Y_{i} \subseteq V(C)$. Let $\mathcal{Y}^{\prime}$ be those sets of $\mathcal{Y}$ which contain vertices of $V(C)$. We define a new partition $\mathcal{Z}$ of $F$ as follows:

$$
\mathcal{Z}=\mathcal{Y} \backslash \mathcal{Y}^{\prime} \cup V(C)
$$


That is, we merge together all sets $Y_{i}$ containing vertices of $V(C)$. All the other sets of $\mathcal{Z}$ coincide with the sets of $\mathcal{Y}$. We have the following statement.

Lemma 2.4. For arbitrary choice of representatives of sets in $\mathcal{Z}$ we have

$$
\operatorname{GPa}^{\mathcal{Y}}(F) \leq \mathrm{GPa}^{\mathcal{Z}}(F)
$$

Proof. Observe that there are three types of sets in $\mathcal{Z}$. First, if $v_{1} \in V(C)$ is a trivial orbit in $G$, then orbits of vertices of the $v_{1}$-rooted tree are sets of $\mathcal{Z}$. Second, if $\left\{v_{1}, \ldots, v_{t}\right\} \subseteq$ $V(C)$ is a non-trivial orbit in $G$, that is if $t \geq 2$, then the $v_{i}$-rooted trees are paths with endvertices $v_{i}$, and sets of vertices of $O_{v_{1}}(F)$ in $\mathcal{Z}$ contain vertices of these $t$ rooted trees which are at the same distance from $C$. Finally, $\mathcal{Z}$ contains $V(C)$. If $Z_{i}$ is a set of $\mathcal{Z}$ of the first type or of the second type and $u, v \in Z_{i}$, then $w_{Z_{i}}(u)=w_{Z_{i}}(v)$. Hence, to prove the statement it suffices to show that

$$
\sum_{Y_{i} \in \mathcal{Y}^{\prime}} w_{Y_{i}}\left(y_{i}\right) \leq w_{V(C)}(z)=\sum_{Y_{i} \in \mathcal{Y}^{\prime}} w_{Y_{i}}(z)
$$

where $z$ is an arbitrary vertex of $V(C)$ and $\mathcal{Y}^{\prime}$ is defined before Lemma 2.4. (Recall that $y_{i}$ is a representative of $Y_{i}$ in $\mathcal{Y}$.)

Thus, let $z \in V(C)$ and let $Y_{i} \in \mathcal{Y}^{\prime}$. In what follows we show that $w_{Y_{i}}\left(y_{i}\right) \leq w_{Y_{i}}(z)$. We distinguish four cases.

Case 1: $\left|Y_{i}\right|=1$. Since $w_{Y_{i}}\left(y_{i}\right)=0 \leq \operatorname{dist}_{F}\left(z, y_{i}\right)=w_{Y_{i}}(z)$, we have $w_{Y_{i}}\left(y_{i}\right) \leq$ $w_{Y_{i}}(z)$.

Case 2: $\left|Y_{i}\right|=2$. let $Y_{i}=\left\{y_{i}, y\right\}$. Then by triangle inequality

$$
w_{Y_{i}}\left(y_{i}\right)=\operatorname{dist}_{F}\left(y_{i}, y\right) \leq \operatorname{dist}_{F}\left(z, y_{i}\right)+\operatorname{dist}_{F}(z, y)=w_{Y_{i}}(z) .
$$

Hence, in the sequel we assume that $\left|Y_{i}\right| \geq 3$. Since $Y_{i}$ is an orbit of vertices of $C$ in $G$, there is a nontrivial rotational automorphism $\alpha$ in $\operatorname{Aut}(G)$ such that $\left\{\alpha^{k}\left(y_{i}\right) \mid k \in \mathbb{N}\right\} \subseteq$ $Y_{i}$. Let $r$ be the biggest order of a rotational automorphism of this type and let $\alpha$ be the corresponding automorphism. Observe that $r \geq 2$. Since $w_{Y_{i}}(u)=w_{Y_{i}}(v)$ for $u, v \in Y_{i}$, we assume that $y_{i}$ is chosen so that $\operatorname{dist}_{F}\left(z, y_{i}\right)$ is smallest possible.

Case 3: $r$ is even. Let $Y_{i}^{\prime}=\left\{\alpha^{k}\left(y_{i}\right) \mid 0 \leq k<r\right\}$. We rename vertices of $Y_{i}^{\prime}$ as $\left\{y^{0}, y^{1}, \ldots, y^{r-1}\right\}$ so that $\operatorname{dist}_{F}\left(y_{i}, y^{k}\right) \leq \operatorname{dist}_{F}\left(y_{i}, y^{k+1}\right)$ whenever $0 \leq k<r-1$. Observe that $y^{0}=y_{i}$, the vertices $y^{2 \ell-1}$ and $y^{2 \ell}$ have the same distance from $y_{i}$ if $1 \leq \ell<$ $r / 2$ and $y^{r-1}$ is the unique vertex of $Y_{i}^{\prime}$ with the largest distance from $y_{i}$. Since $y_{i}$ is the vertex of $Y_{i}$ with the smallest distance from $z$, we have

$$
\operatorname{dist}_{F}\left(y^{2 \ell-1}, y_{i}\right)+\operatorname{dist}_{F}\left(y_{i}, y^{2 \ell}\right)=\operatorname{dist}_{F}\left(y^{2 \ell-1}, z\right)+\operatorname{dist}_{F}\left(z, y^{2 \ell}\right)
$$

for $1 \leq \ell<r / 2$ and also

$$
\operatorname{dist}_{F}\left(y_{i}, y^{r-1}\right)=\operatorname{dist}_{F}\left(y_{i}, z\right)+\operatorname{dist}_{F}\left(z, y^{r-1}\right)=\frac{1}{2}|V(C)| .
$$

Hence, $w_{Y_{i}^{\prime}}\left(y_{i}\right)=w_{Y_{i}^{\prime}}(z)$. If $Y_{i}=Y_{i}^{\prime}$, we are done. Therefore, in the sequel assume that there is also a reflexion $\beta$ such that $\beta\left(Y_{i}^{\prime}\right) \subseteq Y_{i}$ and $\beta\left(Y_{i}^{\prime}\right) \cap Y_{i}^{\prime}=\emptyset$. Then $Y_{i}=Y_{i}^{\prime} \cup \beta\left(Y_{i}^{\prime}\right)$ and $\left|Y_{i}\right|=2 r$. Observe that all the vertices of $\beta\left(Y_{i}^{\prime}\right)$ are obtained from arbitrary one of 
them using $\alpha$. Thus, let $y_{i}^{\beta}$ be a vertex of $\beta\left(Y_{i}^{\prime}\right)$ with the smallest distance from $y_{i}$. Then using the same arguments as above we get

$$
w_{\beta\left(Y_{i}^{\prime}\right)}\left(y_{i}^{\beta}\right)=w_{\beta\left(Y_{i}^{\prime}\right)}\left(y_{i}\right)
$$

Since $w_{\beta\left(Y_{i}^{\prime}\right)}\left(y_{i}^{\beta}\right)=w_{Y_{i}^{\prime}}\left(y_{i}\right)$, we get

$$
w_{Y_{i}}\left(y_{i}\right)=2 w_{Y_{i}^{\prime}}\left(y_{i}\right)
$$

Analogously, if $y_{i}^{\beta z}$ is a vertex of $\beta\left(Y_{i}^{\prime}\right)$ at the smallest distance from $z$, we get

$$
w_{\beta\left(Y_{i}^{\prime}\right)}\left(y_{i}^{\beta z}\right)=w_{\beta\left(Y_{i}^{\prime}\right)}(z)
$$

Since $w_{\beta\left(Y_{i}^{\prime}\right)}\left(y_{i}^{\beta z}\right)=w_{Y_{i}^{\prime}}\left(y_{i}\right)$, we obtain $w_{Y_{i}}\left(y_{i}\right)=w_{Y_{i}}(z)$.

Case 4: $r$ is odd. Let $Y_{i}^{\prime}=\left\{\alpha^{k}\left(y_{i}\right) \mid 0 \leq k<r\right\}$. Then proceeding analogously as in Case 3 one gets

$$
w_{Y_{i}^{\prime}}(z)=w_{Y_{i}^{\prime}}\left(y_{i}\right)+\operatorname{dist}_{F}\left(y_{i}, z\right)
$$

and so $w_{Y_{i}}\left(y_{i}\right) \leq w_{Y_{i}}(z)$ if $Y_{i}=Y_{i}^{\prime}$. Hence, assume that there is a reflexion $\beta$ such that $\beta\left(Y_{i}^{\prime}\right) \subseteq Y_{i}$ and $\beta\left(Y_{i}^{\prime}\right) \cap Y_{i}^{\prime}=\emptyset$. Again, $Y_{i}=Y_{i}^{\prime} \cup \beta\left(Y_{i}^{\prime}\right)$ and $\left|Y_{i}\right|=2 r$, and all the vertices of $\beta\left(Y_{i}^{\prime}\right)$ are obtained from arbitrary one of them using $\alpha$. Thus, let $y_{i}^{\beta}$ be a vertex of $\beta\left(Y_{i}^{\prime}\right)$ with the shortest distance from $y_{i}$. Then analogously as in Case 3 one gets

$$
w_{Y_{i}^{\prime}}\left(y_{i}\right)=w_{\beta\left(Y_{i}^{\prime}\right)}\left(y_{i}^{\beta}\right)=w_{\beta\left(Y_{i}^{\prime}\right)}\left(y_{i}\right)-\operatorname{dist}_{F}\left(y_{i}^{\beta}, y_{i}\right)
$$

and so

$$
w_{Y_{i}}\left(y_{i}\right)=2 w_{Y_{i}^{\prime}}\left(y_{i}\right)+\operatorname{dist}_{F}\left(y_{i}^{\beta}, y_{i}\right) .
$$

Also, let $y_{i}^{\beta z}$ be a vertex of $\beta\left(Y_{i}^{\prime}\right)$ with the shortest distance from $z$. Then analogously as above we get

$$
w_{Y_{i}^{\prime}}\left(y_{i}\right)=w_{\beta\left(Y_{i}^{\prime}\right)}\left(y_{i}^{\beta z}\right)=w_{\beta\left(Y_{i}^{\prime}\right)}(z)-\operatorname{dist}_{F}\left(y_{i}^{\beta z}, z\right)
$$

and so

$$
w_{Y_{i}}(z)=2 w_{Y_{i}^{\prime}}\left(y_{i}\right)+\operatorname{dist}_{F}\left(y_{i}^{\beta z}, z\right)+\operatorname{dist}_{F}\left(y_{i}, z\right)
$$

Since

$$
\operatorname{dist}_{F}\left(y_{i}^{\beta}, y_{i}\right) \leq \operatorname{dist}_{F}\left(y_{i}^{\beta z}, y_{i}\right) \leq \operatorname{dist}_{F}\left(y_{i}^{\beta z}, z\right)+\operatorname{dist}_{F}\left(y_{i}, z\right),
$$

we get $w_{Y_{i}}\left(y_{i}\right) \leq w_{Y_{i}}(z)$.

Finally, we are in a position to prove the last lemma which implies Theorem 1.3. Observe that there is a strict inequality in Lemma 2.5 .

Lemma 2.5. We have

$$
\operatorname{GPa}^{\mathcal{Z}}(F)<\operatorname{GPa}\left(C_{n}\right)
$$

Proof. Analogously as in Lemma 2.3, we prove the statement by a sequence of steps. Let $O^{1}, \ldots, O^{q}$ be all orbits of vertices of $C$ in $G$, such that for every $v \in O^{i}$ the $v$-rooted tree is nontrivial (i.e., it has more than one vertex). Observe that if the $v$-rooted tree is nontrivial in $G$, then the $v$-rooted tree in $F$ is also nontrivial. Assume that $\left|O^{1}\right| \geq\left|O^{2}\right| \geq \cdots \geq\left|O^{q}\right|$. 
We consecutively create unicyclic graphs $F=F^{0}, F^{1}, \ldots, F^{q}=C_{n}$ with partitions $\mathcal{Z}=\mathcal{Z}^{0}, \mathcal{Z}^{1}, \ldots, \mathcal{Z}^{q}$, respectively, and for every $i, 0 \leq i<q$, we show that $\operatorname{GPa}^{\mathcal{Z}^{i}}\left(F^{i}\right)<$ $\mathrm{GPa}^{\mathcal{Z}^{i+1}}\left(F^{i+1}\right)$. The graph $F^{i+1}$ is obtained from $F^{i}$ by moving the vertices of $v$-rooted trees, where $v \in O^{i+1}$, into the unique cycle $C^{i}$ of $F^{i}$. Now we describe the process in detail.

Choose $i, 0 \leq i<q$. For $v \in O^{i+1}$, let $p+1$ be the number of vertices of $v$-rooted tree in $F^{i}$ (or in $G$, since the numbers of vertices of $v$-rooted trees in $G$ and $F$ are the same). By our assumption $p \geq 1$. Orient the cycle $C^{i}$ of $F^{i}$ and for every vertex $u \in V\left(C^{i}\right)$ let $u^{f}$ be the vertex following $u$ on $C^{i}$. Let $v \in O^{i+1}$. Delete the $p$ non-root vertices of the $v$-rooted tree from $F^{i}$ and subdivide the edge $v v^{f}$ exactly $p$ times. Repeat this procedure for all vertices of $O^{i+1}$ and denote by $F^{i+1}$ the resulting unicyclic graph. The partition $\mathcal{Z}^{i+1}$ is exactly the same as $\mathcal{Z}^{i}$, the only exception is that instead of the set $V\left(C^{i}\right)$ and various sets partitioning $O_{v}\left(F^{i}\right)$ for $v \in O^{i+1}$ we have just the set $V\left(C^{i+1}\right)$ in $\mathcal{Z}^{i+1}$. We assume that if a set of $\mathcal{Z}^{i}$ is identical with a set of $\mathcal{Z}^{i+1}$, then they have the same representatives.

Let $Z^{\prime} \in \mathcal{Z}^{i}$ and $Z^{*} \in \mathcal{Z}^{i+1}$ such that $Z^{\prime}=Z^{*}$. Then $Z^{\prime}$ is a collection of vertices of $O_{u}\left(F^{i}\right)$, i.e., of $u$-rooted trees for $u \in O^{j}$, where $j>i+1$. Since the distances between these vertices cannot be shorter in $F^{i+1}$ than in $F^{i}$ (they can be only larger due to the extension of $C^{i}$ to $C^{i+1}$, we have $w_{Z^{\prime}}\left(z^{\prime}\right) \leq w_{Z^{*}}\left(z^{*}\right)$ where $z^{\prime}$ is a representative of $Z^{\prime}$ in $F^{i}$ and $z^{*}$ is a representative of $Z^{*}$ in $F^{i+1}$. Hence, it suffices to check the contribution of $V\left(C^{i+1}\right)$ in $\mathrm{GPa}^{\mathcal{Z}^{i+1}}\left(F^{i+1}\right)$ and in $\mathrm{GPa}^{\mathcal{Z}^{i}}\left(F^{i}\right)$ the contribution of $V\left(C^{i}\right)$ and of the sets of non-root vertices of $v$-rooted trees for $v \in O^{i+1}$. Let $t=\left|O^{i+1}\right|$. Analogously as in the proof of Lemma 2.4 we distinguish four cases. In these cases, we set $c=\left|V\left(C^{i}\right)\right|$. Moreover, by $\delta_{a}$ we denote the parity of $a$. That is $\delta_{a}=1$ if $a$ is odd and $\delta_{a}=0$ if $a$ is even.

Case 1: $t=1$. By Proposition 2.1, $V\left(C^{i}\right)$ contributes $\frac{1}{4}\left(c^{2}-\delta_{c}\right)$ to $\mathrm{GPa}^{\mathcal{Z}^{i}}\left(F^{i}\right)$ and $V\left(C^{i+1}\right)$ contributes $\frac{1}{4}\left((c+p)^{2}-\delta_{c+p}\right)$ to $\mathrm{GPa}^{\mathcal{Z}^{i+1}}\left(F^{i+1}\right)$. Let $O^{i+1}=\left\{v_{1}\right\}$. Denote by $T$ the $v_{1}$-rooted tree in $F^{i}$. Since $T$ is a tree on $p+1$ vertices, the orbits of $T$ contribute to $\mathrm{GPa}^{\mathcal{Z}^{i}}\left(F^{i}\right)$ at most $\frac{1}{4}\left((p+1)^{2}-\delta_{p+1}\right)$ by Theorem 1.2. So

$$
\begin{aligned}
4\left(\mathrm{GPa}^{\mathcal{Z}^{i+1}}\left(F^{i+1}\right)-\mathrm{GPa}^{\mathcal{Z}^{i}}\left(F^{i}\right)\right) & \geq(c+p)^{2}-\delta_{c+p}-c^{2}+\delta_{c}-(p+1)^{2}+\delta_{p+1} \\
& \geq(c+p)^{2}-c^{2}-(p+1)^{2}-1 \\
& =2 p(c-1)-2>0
\end{aligned}
$$

since $c \geq 3$ and $p \geq 1$.

Case 2: $t=2$. In this case the $v$-rooted trees are paths whenever $v \in O^{i+1}$. Since the contribution to $\mathrm{GPa}^{\mathcal{Z}^{i}}\left(F^{i}\right)$ of $j$-th vertices of these paths (i.e., of vertices at distance $j$ from the roots) is at most $j+c / 2+j$, the total contribution of non-root vertices of $v$-rooted trees, $v \in O^{i+1}$, is at most $2\left(\begin{array}{c}p+1 \\ 2\end{array}\right)+\frac{1}{2} c p$. Since the contribution of $V\left(C^{i}\right)$ is $\frac{1}{4}\left(c^{2}-\delta_{c}\right)$ and the contribution of $V\left(C^{i+1}\right)$ is $\frac{1}{4}\left((c+2 p)^{2}-\delta_{c+2 p}\right)$, where $\delta_{c+2 p}=\delta_{c}$, we get

$$
\begin{aligned}
4\left(\mathrm{GPa}^{\mathcal{Z}^{i+1}}\left(F^{i+1}\right)-\mathrm{GPa}^{\mathcal{Z}^{i}}\left(F^{i}\right)\right) & \geq(c+2 p)^{2}-\delta_{c}-c^{2}+\delta_{c}-8\left(\begin{array}{c}
p+1 \\
2
\end{array}\right)-2 c p \\
& \geq(c+2 p)^{2}-c^{2}-4 p^{2}-4 p-2 c p \\
& =2 p(c-2)>0
\end{aligned}
$$

since $c \geq 3$ and $p \geq 1$. 
In the remaining cases we may assume that there is a nontrivial rotational automorphism $\alpha$ of $F^{i}$ such that when $v \in O^{i+1}$ then also $\alpha(v) \in O^{i+1}$. Let $r$ be the biggest order of such a rotational automorphism $\alpha$, and moreover, let $\alpha$ be such that the distance $s$ between $v$ and $\alpha(v)$ is the smallest possible. Then $c=r \cdot s$.

Case 3: $r$ is even. Then $r \geq 2$. Let $v_{0} \in O^{i+1}$. Denote $O^{\prime}=\left\{\alpha^{k}\left(v_{0}\right) \mid 0 \leq k<r\right\}$. First assume that $\left|O^{i+1}\right|=2 r$. Hence there is also a reflexion $\beta$ such that $\beta\left(O^{\prime}\right) \subseteq O^{i+1}$ and $\beta\left(O^{\prime}\right) \cap O^{\prime}=\emptyset$. Let $v_{1} \in O^{i+1}$ such that the distance $t$ between $v_{0}$ and $v_{1}$ is the smallest possible. Then $t \leq s / 2$ and $v_{1} \in \beta\left(O^{\prime}\right)$. Observe that now $t \geq 1$ and $s \geq 2$. Since $c=r s$ is even, the contribution of $V\left(C^{i}\right)$ to $\mathrm{GPa}^{\mathcal{Z}^{i}}\left(F^{i}\right)$ is $\frac{1}{4} r^{2} s^{2}$. The contribution of $V\left(C^{i+1}\right)$ to $\mathrm{GPa}^{\mathcal{Z}^{i+1}}\left(F^{i+1}\right)$ is $\frac{1}{4} r^{2}(s+2 p)^{2}$. Now we calculate the contribution of non-root vertices of $v$-rooted trees when $v \in O^{i+1}$. These trees are paths and the contribution of $j$-th vertices is

$$
\begin{aligned}
& 2(s+2 j)+2(2 s+2 j)+\cdots+2\left(\left(\frac{r}{2}-1\right) s+2 j\right)+\left(\frac{r}{2} s+2 j\right) \\
& \quad+(t+2 j)+(s+t+2 j)+\cdots+\left(\left(\frac{r}{2}-1\right) s+t+2 j\right)+(s-t+2 j) \\
& +(2 s-t+2 j)+\cdots+\left(\left(\frac{r}{2}-1\right) s-t+2 j\right)+\left(\frac{r}{2} s-t+2 j\right) \\
& \quad=4(s+2 j)+4(2 s+2 j)+\cdots+4\left(\left(\frac{r}{2}-1\right) s+2 j\right)+2\left(\frac{r}{2} s+2 j\right)+2 j \\
& \quad=\frac{1}{2} r^{2} s+2 j(2 r-1) .
\end{aligned}
$$

So the contribution of non-root vertices of $v$-rooted trees, $v \in O^{i+1}$, is

$$
\sum_{j=1}^{p}\left(\frac{1}{2} r^{2} s+2 j(2 r-1)\right)=\frac{1}{2} r^{2} s p+\left(p^{2}+p\right)(2 r-1) .
$$

Hence

$$
\begin{aligned}
\mathrm{GPa}^{\mathcal{Z}^{i+1}}\left(F^{i+1}\right)-\mathrm{GPa}^{\mathcal{Z}^{i}}\left(F^{i}\right) \geq & \frac{1}{4} r^{2} s^{2}+r^{2} s p+r^{2} p^{2}-\frac{1}{4} r^{2} s^{2} \\
& -\frac{1}{2} r^{2} s p-2 r p^{2}-2 r p+p^{2}+p \\
= & p^{2}(r-1)^{2}+p\left(r\left(\frac{1}{2} r s-2\right)+1\right)>0
\end{aligned}
$$

since $r \geq 2, s \geq 2$ and $p \geq 1$.

In the case when $\left|O^{i+1}\right|=r$, the contribution of $V\left(C^{i+1}\right)$ is $\frac{1}{4} r^{2}(s+p)^{2}$ and the contribution of $j$-th vertices in $v$-rooted trees, $v \in O^{i+1}$, is

$$
2(s+2 j)+2(2 s+2 j)+\cdots+2\left(\left(\frac{r}{2}-1\right) s+2 j\right)+\left(\frac{r}{2} s+2 j\right)=\frac{1}{4} r^{2} s+2 j(r-1) .
$$

So the contribution of non-root vertices of $v$-rooted trees, $v \in O^{i+1}$, is

$$
\sum_{j=1}^{p}\left(\frac{1}{4} r^{2} s+2 j(r-1)\right)=\frac{1}{4} r^{2} s p+\left(p^{2}+p\right)(r-1) .
$$

Hence

$$
\begin{aligned}
\mathrm{GPa}^{\mathcal{Z}^{i+1}}\left(F^{i+1}\right)-\mathrm{GPa}^{\mathcal{Z}^{i}}\left(F^{i}\right) \geq & \frac{1}{4} r^{2} s^{2}+\frac{1}{2} r^{2} s p+\frac{1}{4} r^{2} p^{2}-\frac{1}{4} r^{2} s^{2} \\
& -\frac{1}{4} r^{2} s p-r p^{2}-r p+p^{2}+p \\
= & p^{2}\left(\frac{r}{2}-1\right)^{2}+p\left(r\left(\frac{1}{4} r s-1\right)+1\right)>0
\end{aligned}
$$


since in this case $r \geq 4, s \geq 1$ and $p \geq 1$.

Case 4: $r$ is odd. Then $r \geq 3$. Let $v_{0} \in O^{i+1}$ and $O^{\prime}=\left\{\alpha^{k}\left(v_{0}\right) \mid 0 \leq k<r\right\}$. First assume that $\left|O^{i+1}\right|=2 r$. Hence there is also a reflexion $\beta$ such that $\beta\left(O^{\prime}\right) \subseteq O^{i+1}$ and $\beta\left(O^{\prime}\right) \cap O^{\prime}=\emptyset$. Let $v_{1} \in O^{i+1}$ such that the distance $t$ between $v_{0}$ and $v_{1}$ is the smallest possible. Then $t \leq s / 2$ and $v_{1} \in \beta\left(O^{\prime}\right)$. The contribution of $V\left(C^{i}\right)$ to $\mathrm{GPa}^{\mathcal{Z}^{i}}\left(F^{i}\right)$ is $\frac{1}{4}\left(r^{2} s^{2}-\delta_{r s}\right)$. The contribution of $V\left(C^{i+1}\right)$ to $\mathrm{GPa}^{\mathcal{Z}^{i+1}}\left(F^{i+1}\right)$ is $\frac{1}{4}\left(r^{2}(s+2 p)^{2}-\right.$ $\left.\delta_{r(s+2 p)}\right)$. Now we calculate the contribution of non-root vertices of $v$-rooted trees when $v \in O^{i+1}$. These trees are paths and the contribution of $j$-th vertices is

$$
\begin{aligned}
& 2(s+2 j)+2(2 s+2 j)+\cdots+2\left(\frac{r-1}{2} s+2 j\right) \\
& \quad+(t+2 j)+(s+t+2 j)+\cdots+\left(\frac{r-1}{2} s+t+2 j\right) \\
& +(s-t+2 j)+(2 s-t+2 j)+\cdots+\left(\frac{r-1}{2} s-t+2 j\right) \\
& \quad=4(s+2 j)+4(2 s+2 j)+\cdots+4\left(\frac{r-1}{2} s+2 j\right)+(t+2 j) \\
& \quad=\frac{1}{2}\left(r^{2}-1\right) s+2 j(2 r-1)+t .
\end{aligned}
$$

So the contribution of non-root vertices of $v$-rooted trees, $v \in O^{i+1}$, is

$$
\begin{aligned}
\sum_{j=1}^{p}\left(\frac{1}{2}\left(r^{2}-1\right) s+2 j(2 r-1)+t\right) & =\frac{1}{2}\left(r^{2}-1\right) s p+\left(p^{2}+p\right)(2 r-1)+p t \\
& \leq \frac{1}{2} r^{2} s p+\left(p^{2}+p\right)(2 r-1)
\end{aligned}
$$

since $-\frac{1}{2} s p+p t \leq 0$. Hence

$$
\begin{aligned}
\mathrm{GPa}^{\mathcal{Z}^{i+1}}\left(F^{i+1}\right)-\mathrm{GPa}^{\mathcal{Z}^{i}}\left(F^{i}\right) \geq & \frac{1}{4} r^{2} s^{2}+r^{2} s p+r^{2} p^{2}-\frac{1}{4} \delta_{r s}-\frac{1}{4} r^{2} s^{2}+\frac{1}{4} \delta_{r s} \\
& -\frac{1}{2} r^{2} s p-2 r p^{2}-2 r p+p^{2}+p \\
= & p^{2}(r-1)^{2}+p\left(r\left(\frac{1}{2} r s-2\right)+1\right)>0
\end{aligned}
$$

since $r \geq 3, s \geq 2$ and $p \geq 1$.

In the case when $\left|O^{i+1}\right|=r$, the contribution of $V\left(C^{i+1}\right)$ to $\mathrm{GPa}^{\mathcal{Z}^{i+1}}\left(F^{i+1}\right)$ is $\frac{1}{4}\left(r^{2}(s+p)^{2}-\delta_{r(s+p)}\right)$ and the contribution of $j$-th vertices in $v$-rooted trees, $v \in O^{i+1}$, is

$$
2(s+2 j)+2(2 s+2 j)+\cdots+2\left(\frac{r-1}{2} s+2 j\right)=\frac{1}{4}\left(r^{2}-1\right) s+2 j(r-1) .
$$

So the contribution of non-root vertices of $v$-rooted trees, $v \in O^{i+1}$, is

$$
\sum_{j=1}^{p}\left(\frac{1}{4}\left(r^{2}-1\right) s+2 j(r-1)\right)=\frac{1}{4}\left(r^{2}-1\right) s p+\left(p^{2}+p\right)(r-1) .
$$

Hence

$$
\begin{aligned}
\mathrm{GPa}^{\mathcal{Z}^{i+1}}\left(F^{i+1}\right)-\mathrm{GPa}^{\mathcal{Z}^{i}}\left(F^{i}\right) \geq & \frac{1}{4} r^{2} s^{2}+ \\
& \frac{1}{2} r^{2} s p+\frac{1}{4} r^{2} p^{2}-\frac{1}{4}-\frac{1}{4} r^{2} s^{2} \\
& -\frac{1}{4}\left(r^{2}-1\right) s p-r p^{2}-r p+p^{2}+p \\
= & p^{2}\left(\frac{r}{2}-1\right)^{2}+p\left(r\left(\frac{1}{4} r s-1\right)+\frac{1}{4} s+1\right)-\frac{1}{4}>0
\end{aligned}
$$

since in this case $r \geq 3, s \geq 1$ and $p \geq 1$. (Observe that the second bracket is at least $\frac{2}{4}$ if $r=3$ and $s=1$.) 


\section{References}

[1] A. R. Ashrafi and M. V. Diudea (eds.), Distance, Symmetry, and Topology in Carbon Nanomaterials, volume 9 of Carbon Materials: Chemistry and Physics, Springer, Cham, 2016, doi:10.1007/978-3-319-31584-3.

[2] A. R. Ashrafi, F. Koorepazan-Moftakhar and M. V. Diudea, Topological symmetry of nanostructures, Fuller. Nanotub. Car. N. 23 (2015), 989-1000, doi:10.1080/1536383x.2015.1057818.

[3] A. R. Ashrafi, F. Koorepazan-Moftakhar, M. V. Diudea and O. Ori, Graovac-Pisanski index of fullerenes and fullerene-like molecules, Fuller. Nanotub. Car. N. 24 (2016), 779-785, doi: $10.1080 / 1536383 \times .2016 .1242483$.

[4] A. R. Ashrafi and H. Shabani, The modified Wiener index of some graph operations, Ars Math. Contemp. 11 (2016), 277-284, doi:10.26493/1855-3974.801.968.

[5] M. Črepnjak, M. Knor, N. Tratnik and P. Žigert Pleteršek, The Graovac-Pisanski index of a connected bipartite graph is an integer number, submitted, arXiv:1709.04189 [math.CO].

[6] M. Črepnjak, N. Tratnik and P. Žigert Pleteršek, Predicting melting points of hydrocarbons by the Graovac-Pisanski index, Fuller. Nanotub. Car. N. 26 (2018), 239-245, doi:10.1080/ $1536383 x .2017 .1386657$.

[7] M. Ghorbani and S. Klavžar, Modified Wiener index via canonical metric representation, and some fullerene patches, Ars Math. Contemp. 11 (2016), 247-254, doi:10.26493/1855-3974. $918.0 \mathrm{~b} 2$.

[8] A. Graovac and T. Pisanski, On the Wiener index of a graph, J. Math. Chem. 8 (1991), 53-62, doi:10.1007/bf01166923.

[9] M. Hakimi-Nezhaad and M. Ghorbani, On the Graovac-Pisanski index, Kragujevac J. Sci. 39 (2017), 91-98, doi:10.5937/kgjsci1739091h.

[10] M. Knor, R. Škrekovski and A. Tepeh, Trees with the maximal value of Graovac-Pisanski index, Appl. Math. Comput. 358 (2019), 287-292, doi:10.1016/j.amc.2019.04.034.

[11] F. Koorepazan-Moftakhar and A. R. Ashrafi, Distance under symmetry, MATCH Commun. Math. Comput. Chem. 74 (2015), 259-272, http://match.pmf.kg.ac.rs/ electronic_versions/Match74/n2/match74n2_259-272.pdf.

[12] F. Koorepazan-Moftakhar and A. R. Ashrafi, Combination of distance and symmetry in some molecular graphs, Appl. Math. Comput. 281 (2016), 223-232, doi:10.1016/j.amc.2016.01.065.

[13] F. Koorepazan-Moftakhar, A. R. Ashrafi and O. Ori, Symmetry groups and Graovac-Pisanski index of some linear polymers, Quasigroups Related Systems 26 (2018), 87-102, http: / / www. quasigroups. eu/contents/download/2018/26_10.pdf.

[14] R. Pinal, Effect of molecular symmetry on melting temperature and solubility, Org. Biomol. Chem. 2 (2004), 2692-2699, doi:10.1039/b407105k.

[15] H. Shabani and A. R. Ashrafi, Symmetry-moderated Wiener index, MATCH Commun. Math. Comput. Chem. 76 (2016), 3-18, http://match.pmf.kg.ac.rs/electronic_ versions/Match76/n1/match76n1_3-18.pdf.

[16] N. Tratnik, The Graovac-Pisanski index of zig-zag tubulenes and the generalized cut method, J. Math. Chem. 55 (2017), 1622-1637, doi:10.1007/s10910-017-0749-5.

[17] N. Tratnik and P. Žigert Pleteršek, The Graovac-Pisanski index of armchair tubulenes, J. Math. Chem. 56 (2018), 1103-1116, doi:10.1007/s10910-017-0846-5.

[18] H. Wiener, Structural determination of paraffin boiling points, J. Am. Chem. Soc. 69 (1947), 17-20, doi:10.1021/ja01193a005. 